Stephen L. Campbell, Jean-Philippe Chancelier and Ramine Nikoukhah

\title{
Modeling and Simulation in Scilab/Scicos with ScicosLab 4.4
}

Second Edition

4) Springer 


\section{Contents}

\section{Part I Scilab}

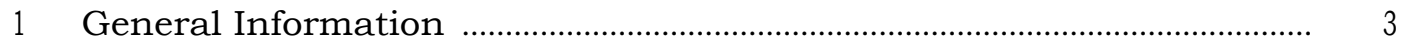

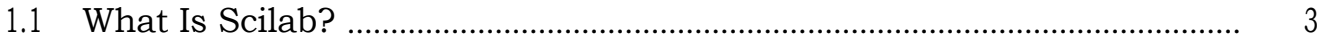

1.2 How to Start? 4

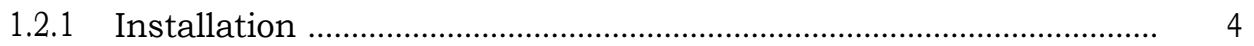

1.2.2 First Steps ...................................................................................... 4

1.2.3 Line Editor ........................................................................................... 5

1.2.4 Documentation ............................................................................. 6

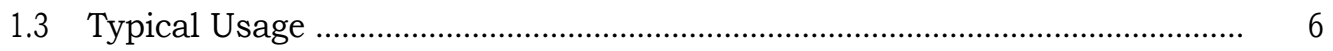

1.4 ScicosLab an the Web .................................................................................... 7

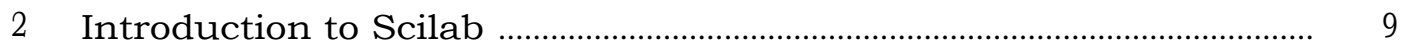

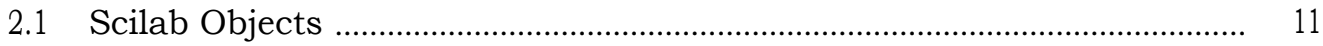

2.1.1 Matrix Construction and Manipulation ……....................................... 12

2.1.2 Strings ................................................................................. 17

2.1.3 Boolean Matrices ............................................................................ 19

2.1.4 Polynomial Matrices ............................................................................... $\quad 20$

2.1.5 Sparse Matrices .................................................................................. 21

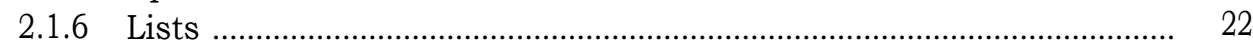

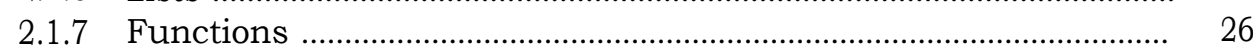

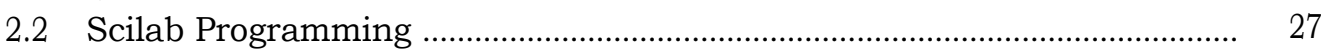

2.2.1 Branching ......................................................................................... 28

2.2 .2 Iterations ............................................................................... 29

2.2.3 Scilab Functions ............................................................................... $\quad 31$

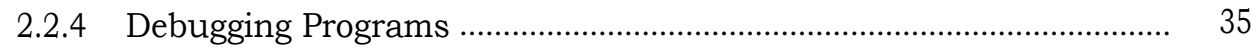

2.3 Input and Output Functions …................................................................. 37

2.3.1 Display of Variables ............................................................................. $\quad 37$

2.3.2 Formatted Input and Output ............................................................ 38

2.3.3 Input Output in Binary Mode .......................................................... 41

2.3.4 Accessing the Host System ……......................................................... 42

2.3.5 Graphical User Interface ............................................................... 43

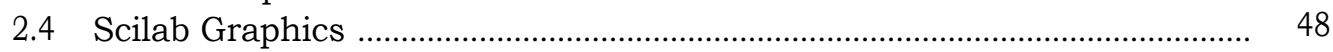

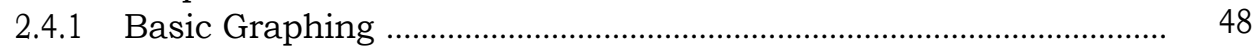

2.4.2 Graphic Tour ................................................................................ 50

2.4.3 Graphics Objects ............................................................................ 53 
2.4.4 Scilab Graphics and Ii5IhiX ................................................................. 56

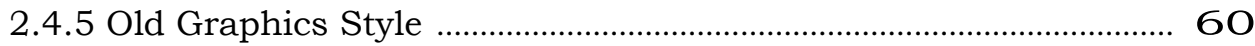

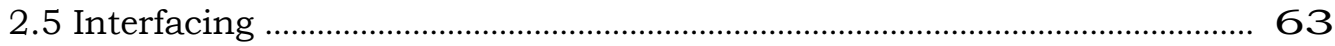

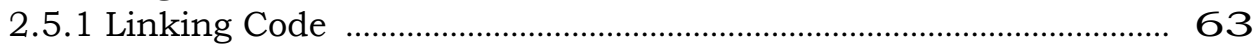

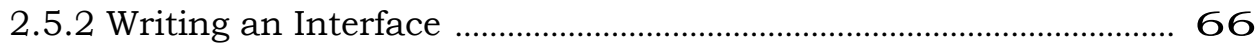

2.5.3 Dynamic Loading ................................................................................... 69

3 Modeling and Simulation in Scilab ........................................................... 73

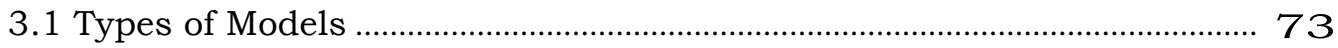

3.1.1 Ordinary Differential Equations ………….......................................... 73

3.1.2 Boundary Value Problems ……………....................................... 74

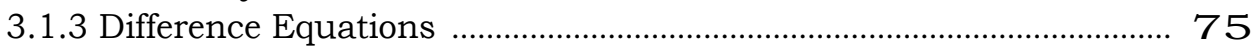

3.1.4 Differential Algebraic Equations ......................................................... 76

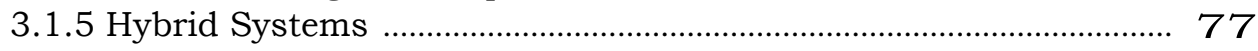

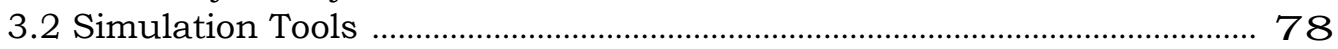

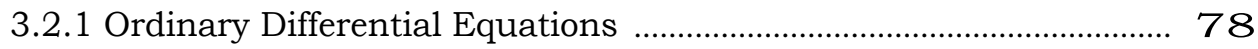

3.2.2 Boundary Value Problems .................................................................... 90

3.2.3 Difference Equations ................................................................................ 95

3.2.4 Differential Algebraic Equations .................................................... 98

3.2.5 Hybrid Systems ................................................................................. 101

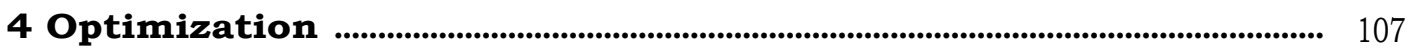

4.1 Comments an Optimization and Solving Nonlinear Equations ..................... 107

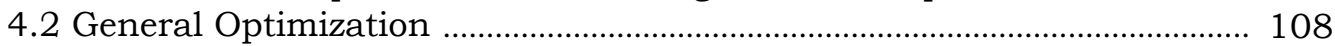

4.3 Solving Nonlinear Equations ……………......................................................... 112

4.4 Nonlinear Least Squares _............................................................................... 113

4.5 Parameter Fitting .......................................................................................... 117

4.6 Linear and Quadratic Programming .............................................................. 119

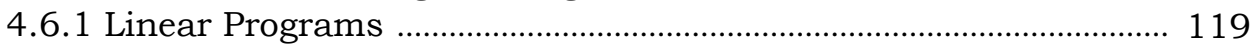

4.6.2 Quadratic Programs ............................................................................ 120

4.6.3 Semidefinite Programs ........................................................................... 120

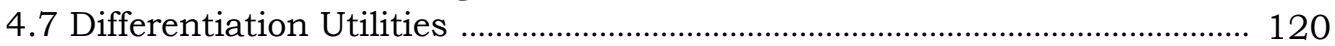

4.7.1 Higher Derivatives .............................................................................. 122

5 Examples ............................................................................................................................... 125

5.1 Modeling and Simulation of an N-Link Pendulum .................................... 125

5.1.1 Equations of Motion of the N-Link Pendulum .................................. 126

5.1.2 Generated Code and Simulation ......................................................... 130

5.1.3 Maple Code ..................................................................................... 133

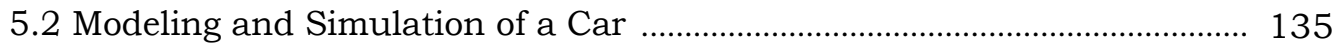

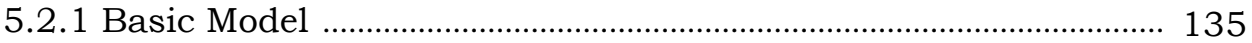

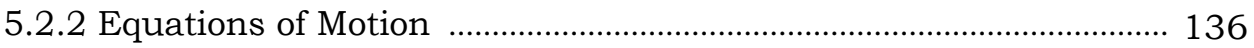

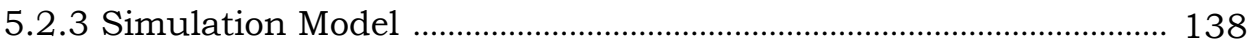

5.2.4 Scilab Implementation …………….................................................... 139

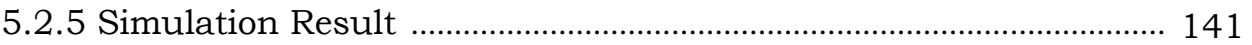

5.3 Open-Loop Control to Swing Up a Pendulum ................................................. 142

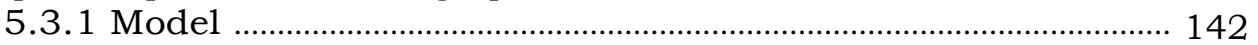

5.3.2 Control Problem Formulation ............................................................ 142

5.3.3 Optimization Problem …………………........................................ 143

5.3.4 Implementation in Scilab .............................................................. 145 
5.4 Parameter Fitting and Implicit Models ....................................................... 147

5.4.1 Mathematical Model ....................................................................... 148

5.4.2 Scilab Implementation .................................................................. 148

Part II Scicos

6 Introduction

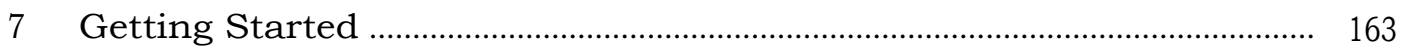

7.1 Construction of a Simple Diagram ………...................................................... 163

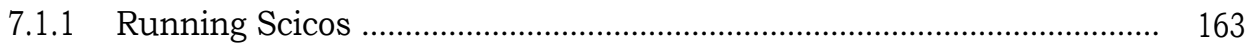

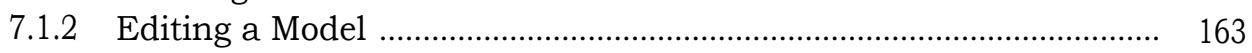

7.1.3 Diagram Simulation ...................................................................... 166

7.1.4 Changing Block Parameters .......................................................... 167

7.2 Symbolic Parameters and Context ................................................................. 170

7.3 Virtual blocks and Hierarchy ........................................................................... 173

7.3.1 Placing a Super Block in a Diagram ............................................. 174

7.3.2 Editing a Super Block .................................................................... 174

7.3.3 Scope of Variables in Super Block Contexts and Masking ................ 176

$7.4 \quad$ Save and Load Operations ............................................................................ 178

7.4.1 Scicos File Formats ...................................................................... 178

7.4.2 Super Block and Palette ...................................................................... 178

7.5 Synchronism and Special Blocks ............................................................. 179

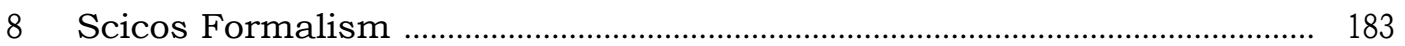

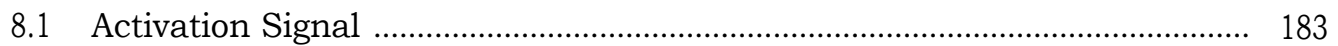

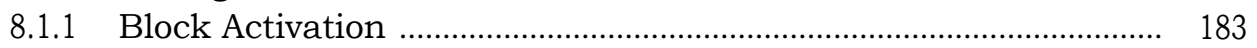

8.1.2 Activation Generation ................................................................... 185

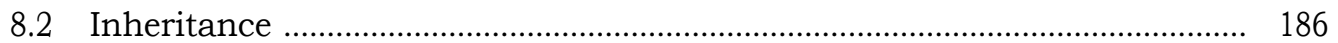

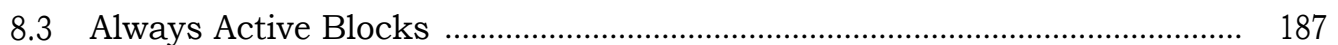

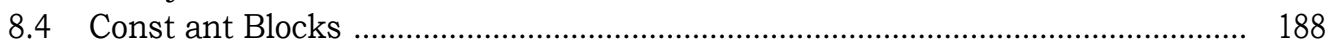

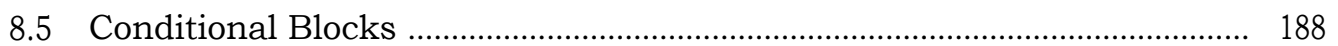

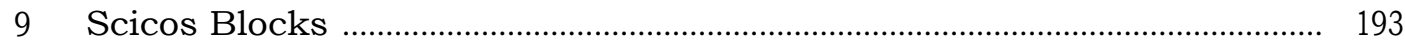

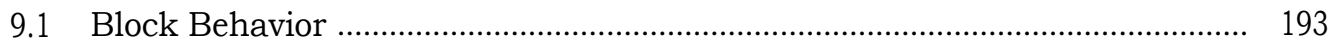

9.1.1 External Activation ............................................................................ 193

9.1.2 Always Activation ........................................................................... 196

9.1.3 Internal Zero-Crossing …………................................................... 196

9.2 Blocks Inside Palettes .............................................................................. 196

9.3 Modifying Block Parameters …………............................................................ 197

9.4 Super Block and Scifunc ………................................................................. 197

9.4.1 Super Blocks ............................................................................... 198

9.4.2 Scifunc ............................................................................................ 198

9.5 Constructing New Basic Blocks ………........................................................ 198

9.5.1 Interfacing Function …................................................................... 199

9.5.2 Computational Function ............................................................ 201

9.5.3 Saving New Blocks ........................................................................... 216

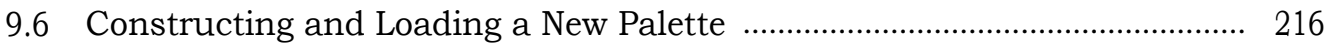


10 Examples and Applications

10.1 Academic examples

10.1.1 Predator Prey Model

10.1.2 Control Application

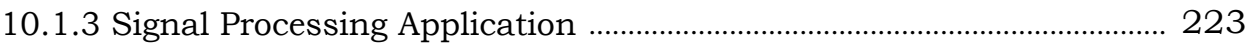

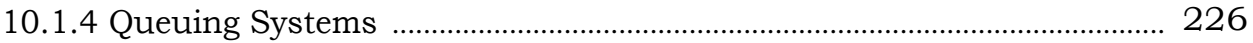

10.1.5 Neuroscience Application …………………………………………... 228

10.1.6 A Fluid Model of TCP-Like Behavior …………..................................... 230

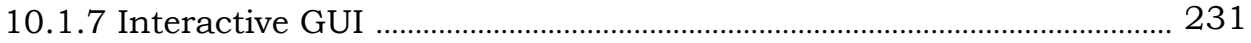

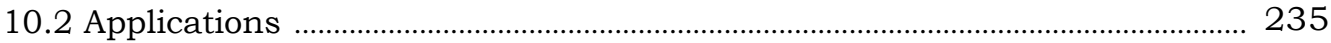

11 Batch Processing in Scilab ………………..................................................................... 239

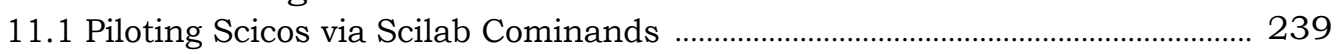

11.1.1 Function scicos im ......................................................................... 240

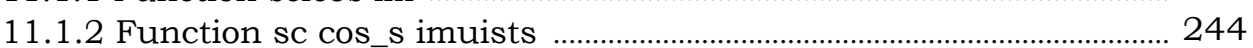

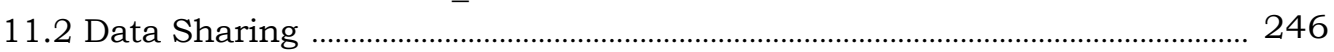

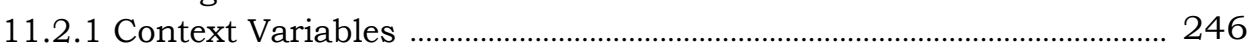

11.2.2 Input/Output Files .................................................................................. 246

11.2.3 Special blocks: From Workspace and To Workspace ................................... 249

11.3 Steady-State Solution and Linearization ……….................................................. 255

11.3.1 Scilab Function steadycos ........................................................................ 257

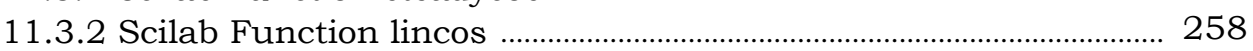

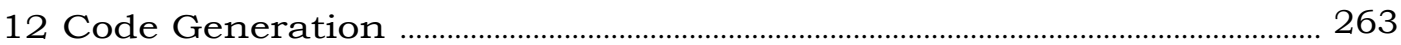

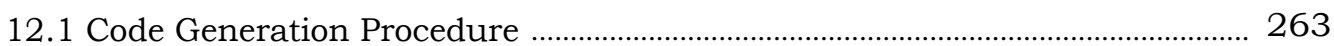

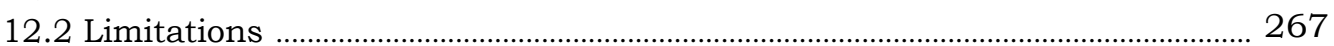

12.2.1 Continuous-Time Activation ........................................................................ 267

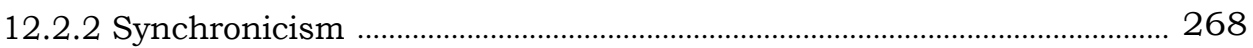

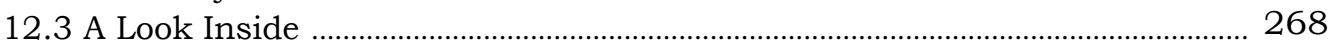

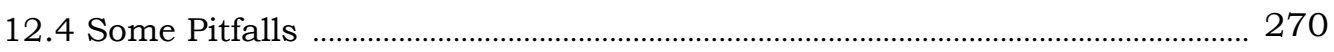

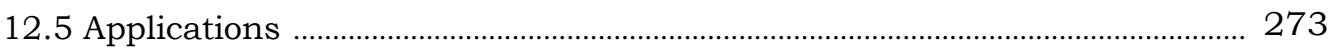

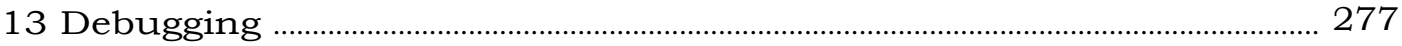

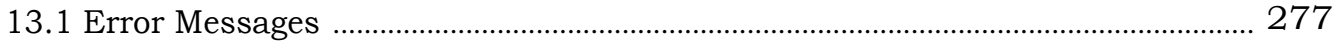

13.1.1 Block Errors ...................................................................................... 277

13.1.2 Errors During Numerical Integration ..................................................... 278

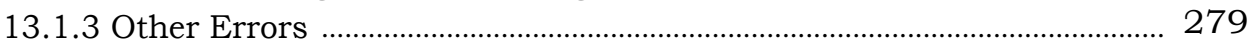

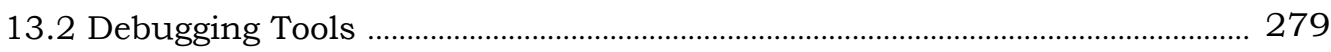

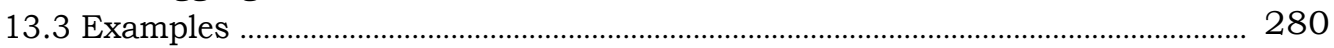

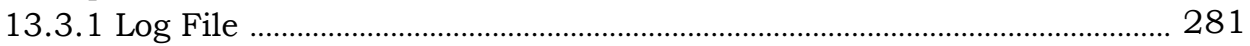

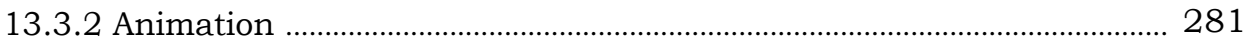

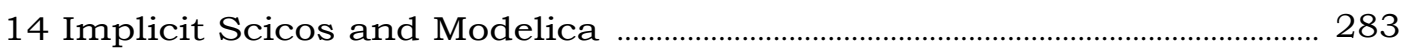

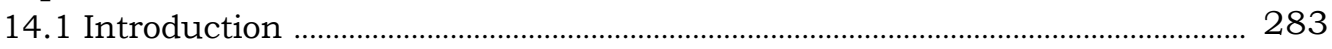

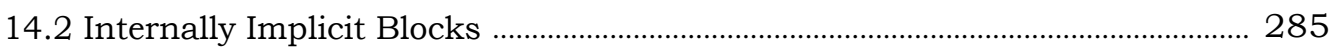

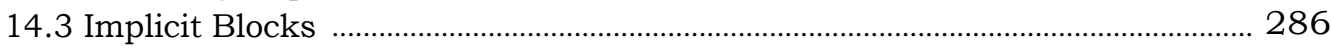

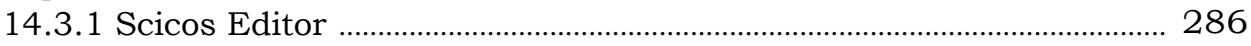

14.3.2 Scicos Compiler ............................................................................................. 286

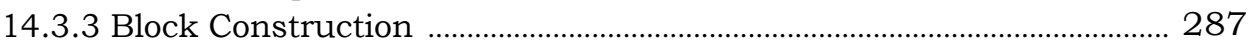

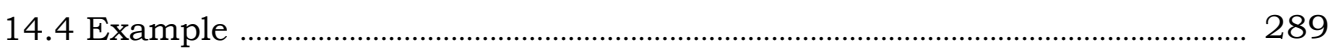


14.5 Scicos Block MBlock

290

14.6 Initialization

292

14.6.1 Example

293

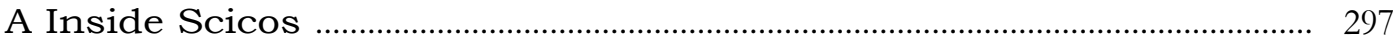

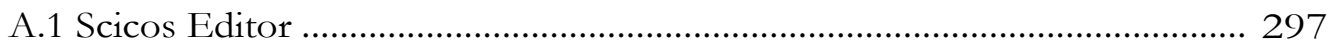

A.1.1 Main Editor Function ................................................................ 297

A.1.2 Structure of scs_m ......................................................................... 300

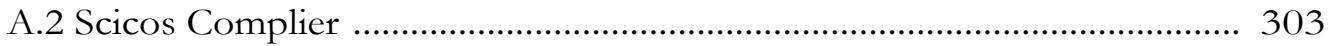

A.2.1 First Compilation Stage ................................................................. 303

A.2.2 Second Compilation Stage ............................................................ 304

A.2.3 Structure of Vocpr ............................................................................ 304

A.2.4 Partial Compilation ..................................................................... 307

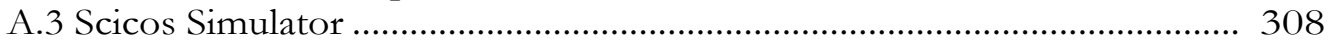

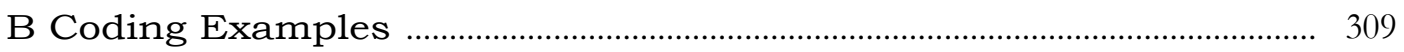

B.1 Scicos Blocks of Type 5 ........................................................................ 309

B.1.1 Type 5 Block for the Bouncing Ball Example ................................ 309

B.1.2 Animation Block for the Cart Pendulum Example ....................... 310

B.2 Animation Program for the Car Example ................................................ 314

B.3 Extraction Program for the Graphic Example .................................. 315

B.4 Maple Code Used for Modeling the N-Link Pendulum ............................... 316

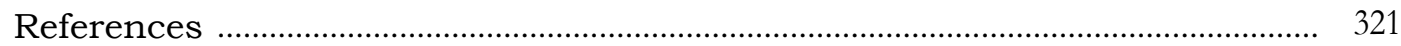

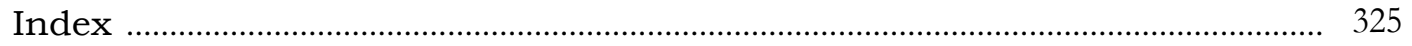

